\title{
Seizures induced by movement: report of a case relieved by operation
}

\author{
M. A. FALCONER, M. V. DRIVER, AND E. A. SERAFETINIDES \\ From the Guy's-Maudsley Neurosurgical Unit, and the Department of Experimental Neurology, \\ Institute of Psychiatry, London, S.E.5
}

Under the title 'seizures induced by movement' Lishman, Symonds, Whitty, and Willison (1962) have recently reported seven cases of a type of epilepsy which they consider rare. These seizures present a fairly uniform pattern, and are characterized by a sudden tonic spasm which may involve the whole body, but more often is localized to one limb or one side of the body. There are no clonic movements or loss of consciousness. Sometimes the movements take an athetoid form. Such seizures usually begin in childhood, last for a few seconds to a minute, but may recur frequently, up to several times daily. They are generally precipitated by sudden movement, and are more likely to occur after a period of rest. Movements of the legs initiate them more often than do movements of the arms, and startle sometimes seems important. Often a state of emotional tension or anxiety precedes them, while conversely mental relaxation or the slow initiation of movement tend to reduce them. Often also a sensory aura briefly precedes the spasm. Sometimes attacks can be voluntarily induced, provided the provoking movement is abrupt and forceful. All the authors' reported cases were males. Lishman et al. (1962) could find only two other cases reported in the literature in sufficient detail to be included in their group (Pitha, 1938; Michaux and Granier, 1945), although Gowers (1901) had earlier discussed three cases in which 'attacks can be brought on by voluntary motion'. They therefore included these two cases in their analysis, and argued that the attacks were epileptic. In addition to the clinical evidence, they pointed out that there are often E.E.G. abnormalities either in the resting record or on overbreathing, when the abnormalities take the form of a build-up of paroxysmal slow activity and/or the emergence of spikes. In six of their seven personal cases anticonvulsant drugs reduced the frequency and severity of attacks and in one abolished them. Lishman et al. could not decide where these attacks arose and speculated whether they could result from some lesion or

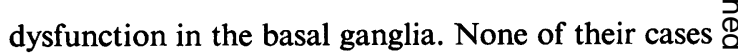
was submitted to surgical exploration.

We therefore wish to report a case which of clinical and E.E.G. grounds conformed to the? seizure pattern described above; the patient appears $\vec{\omega}$ to have been completely relieved by removal of a cicatrix situated on the superior border of one $\overline{3}$ hemisphere immediately in front of the primary motor cortex.

\section{CASE HISTORY}

A 35-year-old medical man, who referred himself, had complained of frequent stereotyped sensorimotor seizures which were crippling his social and professional life. came of a healthy family and his birth had been normat He had had no antecedent serious diseases or heq injuries. Although born in London he had lived for time in the tropics.

The account he gave to us of his illness was that his first attack at the age of 28 years had been a focal convulsive seizure involving the right leg followed within a few seconds by a period of confusion which lasted a few hours. He was investigated in hospital elsewhere with $\overline{\bar{o}}$ negative results, diagnosed as having 'idiopathic epilepsy', ڤै and placed on anticonvulsant drugs, which he had taken $\stackrel{D}{D}$ in various combinations ever since. Radiographs of the skull, chest, and limbs (for cysticercosis) were normal.

A year later his attacks returned and thereafter followed a consistent pattern. At first they occurred once or twice a day, but over the years had increased in frequency until on admission he was averaging six to eight a day, but sometimes having as many as 30 or 40 attacks a day. There was a constant feeling of tension in $:$ the right quadriceps which would increase just before a 3 seizure, and afterwards disappear for a short period. The actual seizure itself was characterized by sudden stiffening of the right leg, followed immediately by arching of the trunk in a convex manner to the right, and by $ㅇ$ abduction of the right shoulder with extension of the $D$ elbow. Such a seizure would last for 10 to 20 seconds, but was not associated with loss of consciousness, inability to speak, or impairment of movement of the right $\mathrm{G}$ wrist or fingers. Occasionally if the attack was severe he $N$ said his consciousness was clouded, but he could always $\mathrm{N}$ call for help. He had never been incontinent nor had he 
bitten his tongue or lip. In some attacks he had fallen over to the left side. He had tried all manner of anticonvulsant drugs but without benefit, and his current medication was Mysoline $250 \mathrm{mg}$. four times daily, Epanutin $100 \mathrm{mg}$. three times daily, phenobarbitone $30 \mathrm{mg}$. three times daily, and $350 \mathrm{mg}$. sodium amytal at night time.

Many attacks appeared to be spontaneous, but over the years he had noticed certain precipitating factors. On waking from sleep he would have an attack which he ascribed to stretching out movements. During the day, mental stress or emotional embarrassment would increase their frequency. Whenever he felt the tension rise in his thigh he could either accelerate or defer the attack. To do the former he would suddenly tense his thigh or kick his knee, and an attack would start forthwith. To defer an attack he would voluntarily try to relax his quadriceps, but such relaxation was only effective for 10 to 15 minutes.
Once an attack was over, he could usually rely on a period of freedom from attacks for three to four hours, and he had learnt to modify his life accordingly. Thus if he were interviewing patients, he would retire to an adjacent room or closet, and induce a seizure. However, the frequency of attacks was such that he had given up driving a car and his professional and social life was gravely jeopardized.

In the past he had been investigated at several different centres, some of which made their records available to us. He had undergone numerous neurological examinations, all with negative findings. Skull radiographs, air encephalograms, and cerebral angiograms had been reported normal on several occasions. Numerous E.E.G. examinations had been carried out, usually with negative findings, although occasionally bilateral slow activity at about $4 \mathrm{c} / \mathrm{s}$ had been observed, sometimes more evident in the left frontal region. On one occasion a relative

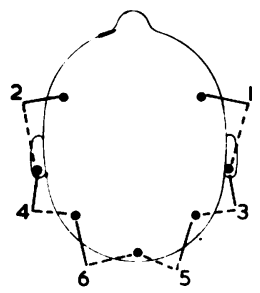

SOHVL

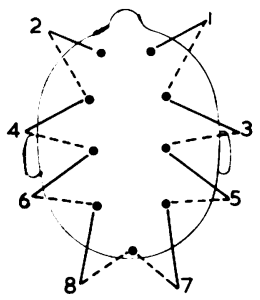

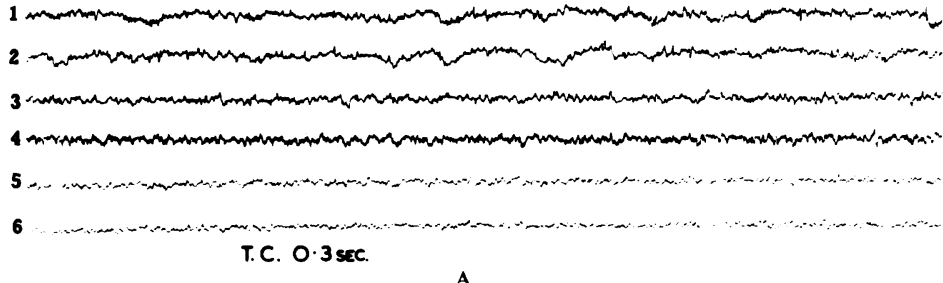

18.4.57.

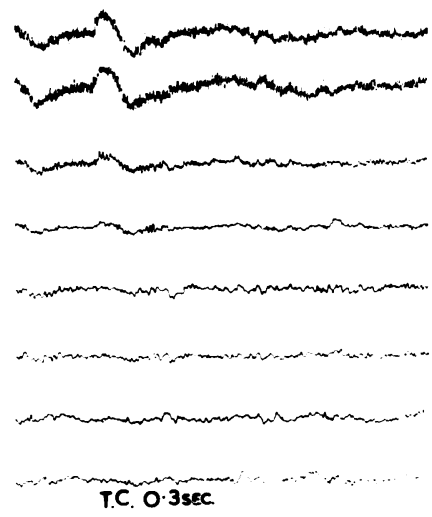

B

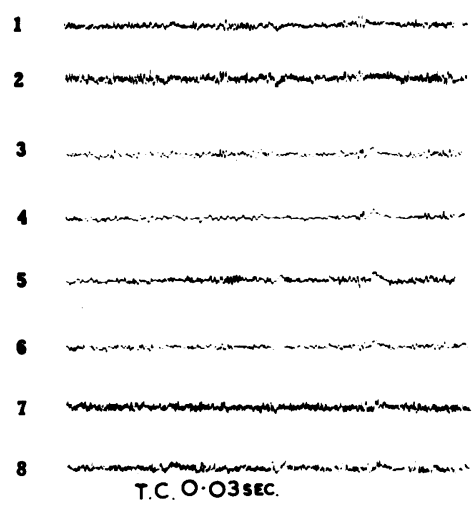

C

FIG. 1. Pre-operative E.E.G.

A November 1955, three days after the last seizure. Bilateral 4-7 c/s waves present in the temporal areas (channels 3 and 4). This activity, which could be recorded over both fronto-temporal regions, was symmetrical on this occasion.

B April 1957, one day after the last seizure. Subject awake: slight relative reduction of fast activity in the left central area (channels 4 and 6, compare with 3 and 5 on the right).

C Continuation of same record as $B$, but subject now asleep following intravenous thiopentone; a similar but more prominent asymmetry of fast activity. 
reduction of spontaneous and induced fast rhythm was observed in the left central area (Fig. 1). Spiking activity had never been noted. Seizures activated by using first Megimide (bemegride) and secondly Metrazol (leptazol) intravenously had been studied four years previously, but with negative results. During the Metrazol activation an attack akin to his habitual seizures occurred, but without an associated alteration in the E.E.G. record. A feature of all these earlier case notes was the consistency with which the patient had described his seizures, and the consistency with which other doctors observing his seizures had also described them. However, several descriptions by lay people seemed to indicate that he had had a number of major convulsive (grand mal) seizures, and the records of one hospital indicated that, after admission and following the abrupt withdrawal of all drugs, he had exhibited a marked confusional state for several days. An exploratory craniotomy on the left side had been considered at one hospital, but declined for lack of positive data.
Our own neurological examination, studies of the $\bar{Z}$ cerebrospinal fluid, skull radiographs, air encephalo- $\stackrel{\mathbb{D}}{\subset}$ grams, and left carotid angiograms were likewise negative. The patient was of high intelligence, right-handed, but $\stackrel{\mathscr{Q}}{\subseteq}$ understandably worried. The routine E.E.G. examination $\bar{\theta}$ was again negative, even though the patient had a 7 spontaneous seizure during it. A further seizure was induced by the slow intravenous injection of $5 \%$ Metrazol. The initial event (as recorded by a ciné camera) was opening the eyes, which appeared to glance quickly to? the left, followed by the assumption of a tonic posture of the right arm with abducted shoulder, Then the head and $\stackrel{5}{S}$ eyes turned to the right and a generalized seizure de- 0 veloped. The camera, however, did not give an adequate 듬 view of the legs. The E.E.G. recorded at the same time $\overline{\bar{c}}$ showed left central changes, including the development of $\vec{\Phi}$ a prominent $20 \mathrm{c} / \mathrm{s}$ rhythm which rapidly slowed to $9 \mathrm{c} / \mathrm{s}$ (Fig. 2). The fit pattern and the E.E.G. phenomena ${ }^{\circ}$ were interpreted as indicating a probable origin of the $\overrightarrow{0}$ seizure in the left hemisphere.
16.61 .0075

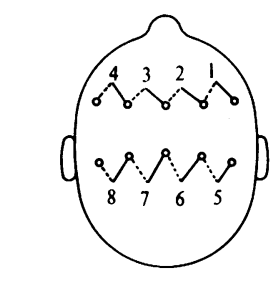

TC $0.3 \mathrm{sec}$ HF Off $\mid 75 \mu \mathrm{v}$

DRUG INDUCED

SEIZURE

(METRAZOL)

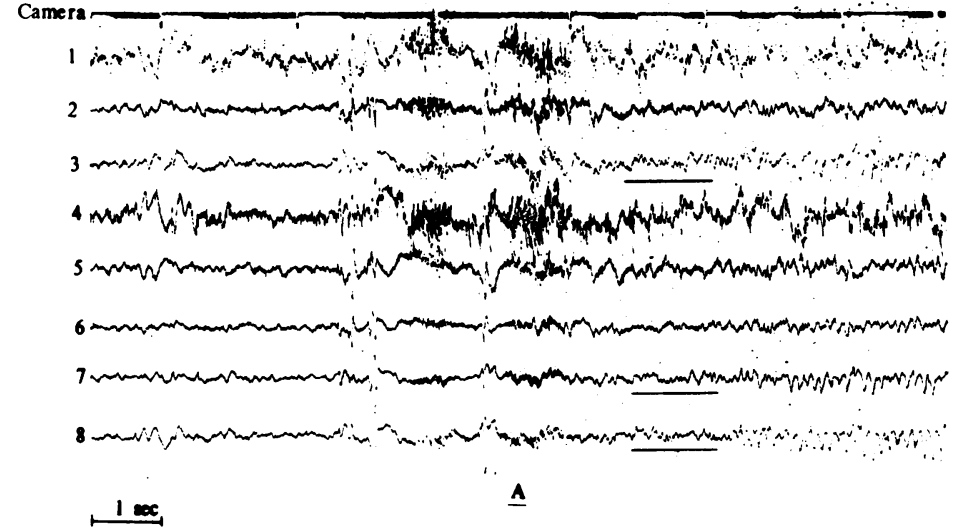

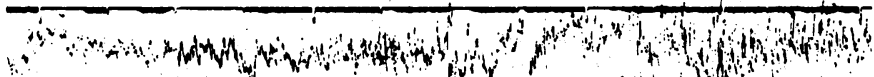

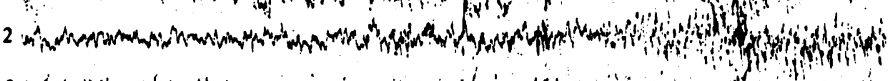
$3 \%$,

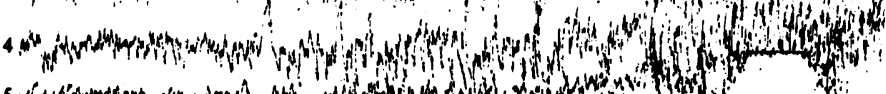

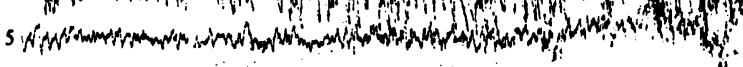

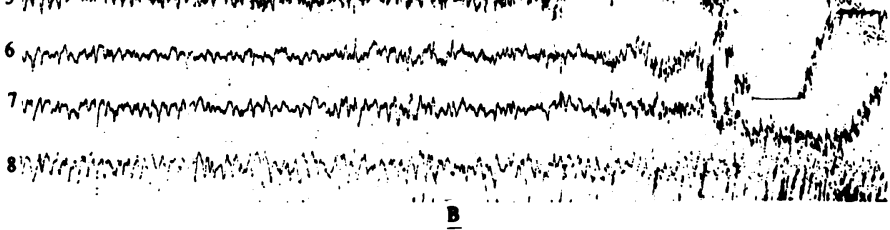

FIG. 2. February 1961, Metrazol-induced seizure.

A After a period of bilateral 'suppression' a low-voltage fast rhythm appears on the left (channels 3, 7, and 8, underlined $)$. This is quickly replaced by a slower rhythm $(9 \mathrm{c} / \mathrm{s})$ which is seen most clearly in channel 8 .

B Immediately after A: the lateralized rhythm continues (compare channels 8 and 5). The artefacts at the end are related to the turning of the head to the right shortly before the onset of the generalized seizure. 
OPERATION In view of the socially and professionally crippling nature of the patient's seizures, an exploratory left-sided craniotomy was recommended and was carried out in February 1961 under local analgesia. The area exposed included the central part of the left cerebral hemisphere up to and including its superior border (Fig. 3). A dense cortical scar $1.5 \mathrm{~cm}$. from before back by $0.5 \mathrm{~cm}$. wide was then observed at the superior border of the hemisphere connected to the overlying dura by adhesions which were severed. This scar, which is seen between points $\mathbf{P}$ and $\mathbf{B}$ in Fig. 3, lay $1 \mathrm{~cm}$. in front of the point at which a large cerebral vein entered the superior longitudinal sinus and probably $2 \mathrm{~cm}$. in front of the Rolandic (central) fissure.

Detailed electrocorticographic (E.Co.G.) and electrical stimulation studies were then carried out, and the results may be summarized as follows. No spikes or abnormal discharges appeared in the resting E.Co.G. record (Fig. 4A) but normal high-voltage rhythmic fast activity at mainly 20 to $24 \mathrm{c} / \mathrm{s}$ was found in all areas. Systematic monopolar electrical stimulation of various points of the exposed cortex, using square-topped anodal pulses of 3 volts, 50 cycles, and $3 \mathrm{msec}$. duration, was then carried out. Only points at which a positive clinical response was elicited are marked in the photograph and these are indicated by alphabetical letters placed in the order in which they were first stimulated. Stimulation of the scar itself had no clinical or electrical effect. Points A, B, E, F, $\mathrm{G}, \mathrm{H}$, and I produced motor movements, the lower ones involving the right face or arm, while only point I produced movements of the right lower limb and then abduction of the hip. Points $\mathrm{C}, \mathrm{J}, \mathrm{K} ; \mathrm{L}, \mathrm{M}, \mathrm{N}$, and $\mathrm{O}$ produced sensory phenomena, the lower ones involving the right arm and the upper the right leg. It was therefore concluded that the Rolandic fissure lay between these two lines of stimulation. Point $P$, just anterior to the scar, produced an increase of tension in the right thigh.

Point D was the critical one. It lay on the mesial side of the scar about $1 \mathrm{~cm}$. deep within the great longitudinal fissure. It was stimulated on four occasions interspaced between the other stimulations without the patient being aware which point was being stimulated. Yet on each occasion the patient remarked that we had duplicated the exact sensation in his right thigh, and on two of these four occasions extension of the right knee occurred with abduction of the right shoulder. All stimulations were monitored by electrocorticography, those at points giving simple sensory or motor phenomena leading to strictly localized after-discharges or none at all. Stimulations of point $\mathrm{D}$, however, led to prominent afterdischarges involving areas on both sides of the scar, the Rolandic surface being affected before the frontal-mesial (Fig. 4B). These after-discharges occurred at the same time as the clinical seizure phenomena. There was no recording electrode in place at point $\mathrm{D}$, but presumably the after-discharges just mentioned began at this point and were transmitted first to the region of electrode 6 (where they were associated with a feeling of tension) followed within five seconds by similar activity at electrode 16 (when the movement occurred).

Next $8 \mathrm{ml}$. of $0.5 \%$ Xylocaine (lignocaine) was injected beneath the scar and around point $D$. An observer (E.A.S.) then reported that there was no weakness in the right leg, elbow, forearm, or hand, but slight weakness

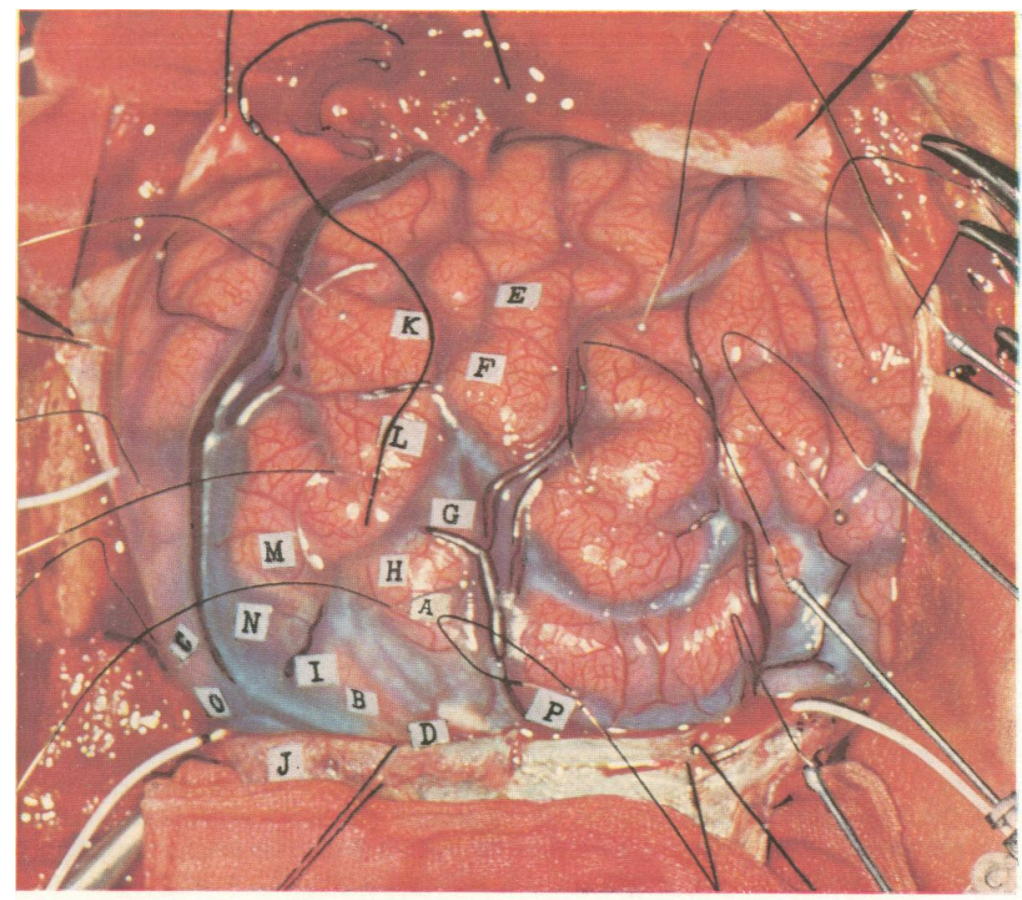

FIG. 3. Operative photograph viewed from the vertex showing lettered points at which positive responses to electrical stimulation were obtained. The cicatrix lies between points $P$ and $B$. Points $D$ and $J$ (represented for convenience on the surface) lay within the great longitudinal fissure. Certain of the recording electrodes can be seen. The magnification is approximately two-thirds. The vertex is at the Dwer margin of the photograph. 


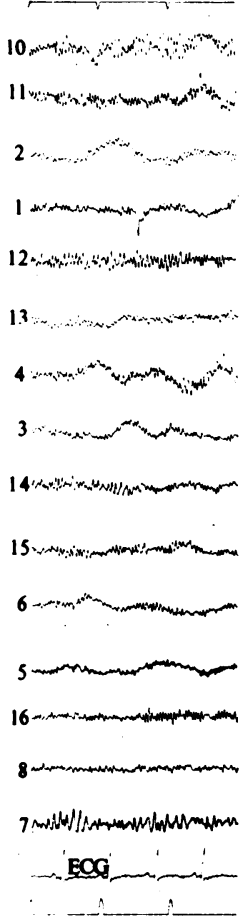

$\mathbf{A}$

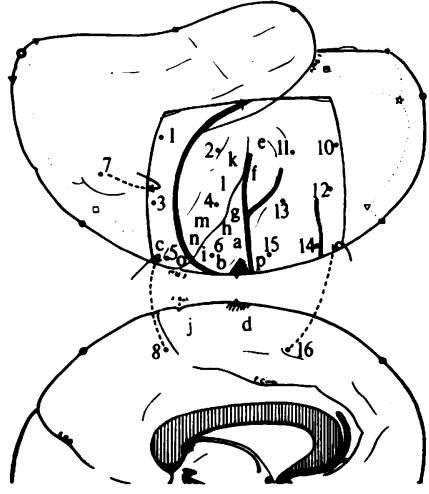

Unipolar, 'average' reference electrode ECoG 164 TCO.1 sec HF Off | $300 \mu \mathrm{v}$
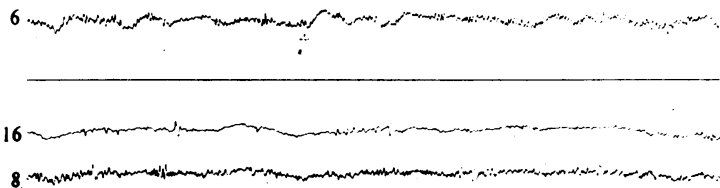

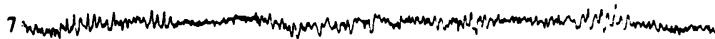

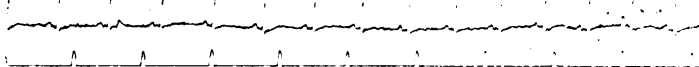

C

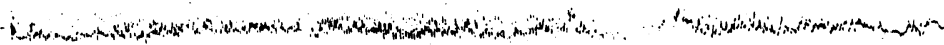
fostimulation ends (point 'd')

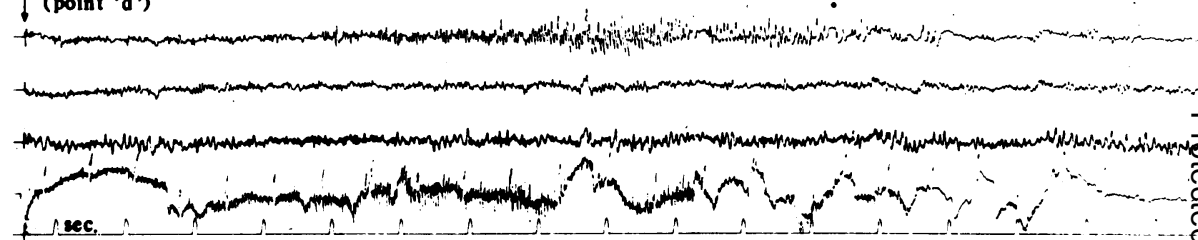

B

FIG. 4. February 1961, electrocorticogram (E.Co.G.). Patient alert and cooperative. The letters indicate points giving clinical responses on electrical stimulation. Electrodes 7, 8, and 16 are attached to insulated flexible wires passed unde. the edge of the craniotomy, 7 to the parieto-occipital convexity, 8 to the mesial parietal area, and 16 to the mesial frontaA. The remaining electrodes are on the exposed cortex (see also Fig. 3).

A Resting E.Co.G.: fast activity in all channels, alpha rhythm at electrode 7.

B Immediately after electrical stimulation of point $d$. A prominent after-discharge appears at electrode 6, associated with a feeling of tension in the right thigh, and is followed in a few seconds by the development of a similar discharge at 16 which is associated with the motor component of the seizure. The after-discharges cease simultaneously about 13 sec. after the end of stimulation, leaving relative 'suppression' at the two electrodes affected (compare with A). Electrodes 8 and 7 (and others not included in this excerpt) show no change following the stimulation. The muscle artefacts in the E.Co.G. are related to the limb movement during the induced seizure. The pen of electrode 5 was switched off in both $B$ and $C$.

C Three minutes after the injection of $8 \mathrm{ml} .0 .5 \%$ Xylocaine beneath the scar and around point d: disappearance of rhythmic fast activity from electrode 16 with development of a few sharp waves. There is a slight fall-out of fast activity from 6 but no change elsewhere.

of the right shoulder. The E.Co.G. (Fig. 4C) showed a reduction in the normal fast activity in the injected region, with the appearance of some sharp waves and spikes. Electrical stimulation of point $D$ now gave no clinical or E.E.G response. The patient, questioned about his sensations, remarked that there was some sensibility in the right arm but that it had disappeared from the leg. The cortical scar was then excised, after which testing of muscle power indicated some weakness of the right shoulder, but not of any other part of the right upper limb. No post-excision E.Co.G. was performed. The craniotomy was closed under local analgesia, and the patient returned to the ward conscious and in good condition.

PATHOLOGY Dr. J. B. Brierley reported that the specimen measured about $1 \mathrm{c} . \mathrm{cm}$., was fibrous to the touch, and resistant to the knife.

'The microscopic sections show an irregular band of apparently normal cortex limited on its deeper aspect by the plane of surgical section which is nowhere deeper than $G$ the junction of the second and third cortical layers. The $N$ cortex is overlaid by thick collagenous tissue, presumably meningeal, which penetrates the cortex here and there 


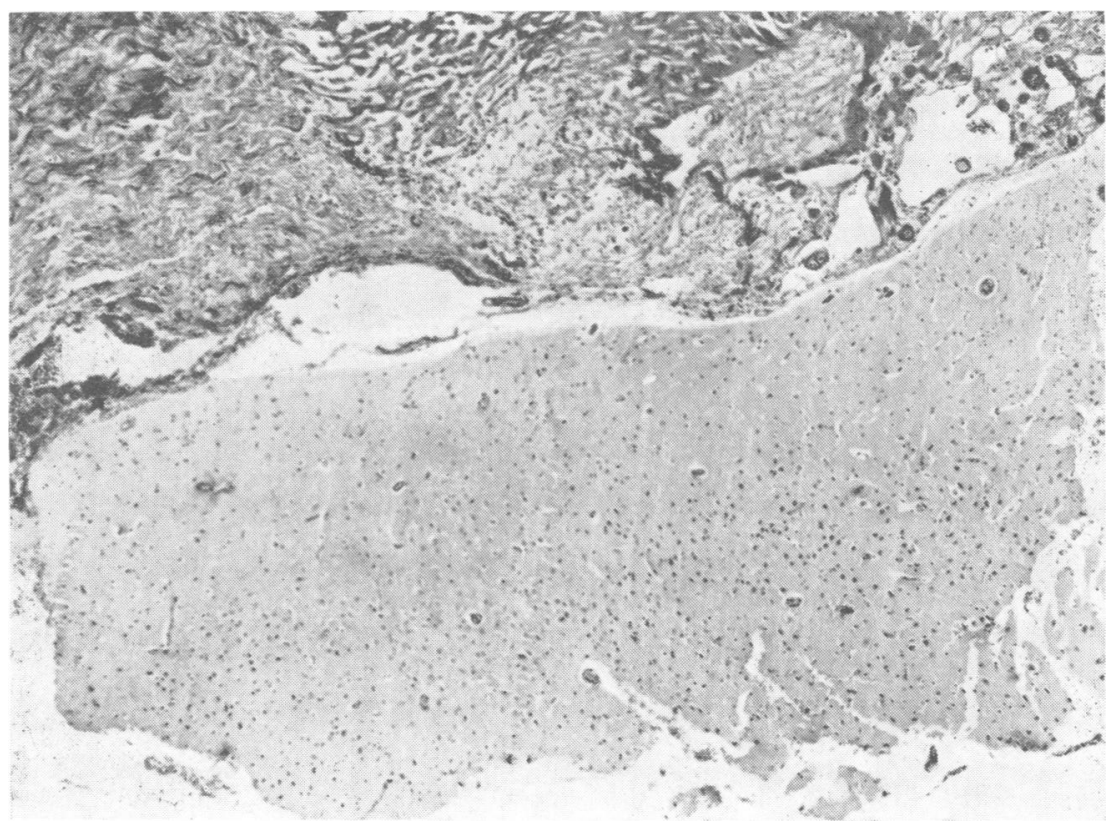

FIG. 5.

Biopsy specimen.

$A$ sheet of fibrous tissue lies on the cortical surface, the outer layers of which show mild gliosis. (Iron haematoxylin and Van Gieson's stain $\times 50$.)

and elsewhere is separated from it by a thin layer of recent haemorrhage (Fig. 5)'.

The case history gave no indications of the aetiology of the lesion.

POST-OPERATIVE PROGRESS On the second post-operative day the patient had three focal motor seizures each characterized by twitching of the right face and right arm, followed by dysphasia. We regarded these as 'neighbourhood seizures' due to oedema of brain tissue at the site of excision. At first he exhibited slight weakness of the right shoulder and elbow, but these features had disappeared at the time of his discharge from hospital. An E.E.G. on the twelfth post-operative day showed no epileptic features. He was prescribed Epanutin, $100 \mathrm{mg}$. thrice daily, and phenobarbitone, $30 \mathrm{mg}$. twice daily.

He was last seen 24 months after operation, and he reported that since returning home he had been completely free of seizures and of the feeling of tension in the right thigh. He was working efficiently at his practice and undertaking normal social activities. He was still taking Epanutin but not phenobarbitone regularly, and he was reluctant to give this up as he regarded it as an insurance against further attacks and indeed had developed the habit of taking it. There were no abnormal neurological signs and an E.E.G. record was normal.

\section{DISCUSSION}

This case raises several points for consideration. First, it fulfils all the criteria described by Lishman, et al. (1962) as characteristic of 'seizures induced by movement'. Secondly the responsible lesion appears to have been a small cicatrix situated on the superior border of the hemisphere $2 \mathrm{~cm}$. in front of the line of the Rolandic (central) fissure. Thirdly, this lesion in provoking epilepsy appears in some way to have involved the supplementary motor area of Penfield and Jasper (1954). Fourthly, our case is an instance of reflex epilepsy, and the question arises as to how the sudden movement induced the seizure. And fifthly, it raises the problem of how other similar cases can be recognized.

The criteria laid down by Lishman and his colleagues have already been considered in the introduction. From our one case we of course cannot tell whether these cases form a homogeneous aetiological group or not, but their characteristics at any rate warrant their being considered a separate group. Lishman and his colleagues felt that these cases were rare, and stressed the dearth of evidence on significant pathological changes in their own and comparable cases in the literature. They concluded that the attacks were a form of reflex epilepsy, and speculated whether some central mechanism involving the basal ganglia could be responsible. They did not consider whether a cortical lesion or mechanism could underlie such seizures. One respect in which our case differed from theirs is that in our patient the seizures appeared uncontrollable by medication, but this is scarcely a critical distinguishing feature.

The complete relief of seizures after excision of 
the cortical scar suggests that the scar itself was the epileptogenic agent, and raises the question of a cortical mechanism being responsible for such seizures as opposed to the mechanism involving the basal ganglia hitherto considered. It also raises the possibility that other such cases might have similar lesions or lesions in similar sites also amenable to surgery if anticonvulsant drugs failed. The operative treatment in our patient was a simple excision of a scar leaving intact the adjacent area of normal-looking cortex from which the patient's actual aura was evoked. We realize that the follow-up period of only 24 months is still short, but the results to date support our expressed view (Falconer, Driver, and Serafetinides, 1962) that in surgery for epilepsy it is more important to excise a visible lesion than normal-looking but firing cortex. Although the pathological lesion may be, as in our case, inert to electrical stimulation and electrocorticography, it appears to act as the triggering mechanism influencing both adjacent areas and areas at a distance.

The fact that the region from which the patient's aura and seizures were consistently evoked by electrical stimulation lay within the great longitudinal fissure a little anterior to the Rolandic fissure suggests that the region chiefly influenced by the scar was the supplementary motor and sensory area (Penfield and Jasper, 1954). The type of tonic posturing exhibited by our patient as well as the sensory aura are in keeping with such a localization, as is also the fact that there was no permanent weakness after ablation of the scar. This localization could also explain the E.E.G. findings, for, as the supplementary motor and sensory area lies within the great longitudinal fissure, it is possible that spiking limited to these regions would not be picked up by surface E.E.G. leads, and even the electrical activity developing during the course of a localized seizure might remain undetected.

The apparent reflex nature of the epilepsy in our patient raises intriguing but as yet insoluble problems. The actual seizure could be induced by sudden deliberate movements of the right quadriceps muscle or by spontaneous movements, as in stretching out the limbs during the act of waking in the morning. It could also occur spontaneously, and after a fit there was a refractory period, during which the stimulus was ineffective. Similar observations have been made in two other cases of reflex epilepsy reported from this Unit. In one patient the visual stimulus induced by the sight of a safety pin was the operative factor (Mitchell, Falconer, and Hill, 1954) and in the other a tactile stimulus to the face could evoke a fit (Goldie and Green, 1959; James, 1959). Comparable observations have also been made in self-induced photoconvulsive epilepsy (Sherwood, 1962).

The sensory aura in our patient is worthy of note, for many if not most of Lishman's cases also show a sensory aura. Penfield and Welch (1951) and Penfield and Jasper (1954) draw attention to the presence of sensory auras in seizures arising in the supplementary motor and sensory area. It is interesting that in our present case the sensory aura, a feeling of tension in the right quadriceps muscle, did not 'march' but simply increased in degree until the sudden movement initiated the motor seizure. This lack of marching suggests that the 'trigger points' for both the sensory and the motor components of the seizure were closely linked, and that the bombardment of the 'sensory point' by the neuronal discharges responsible for the sensory aura had to reach a critical level, augmenting the feeling of tension, before the sudden movement of the quadriceps could be effective.

In view of the lack of a proper understanding of the mechanisms of epilepsy, it is perhaps unwise to speculate more widely as to how this particular form of epilepsy is evoked. A more practical questionp is to consider its recognition, and in particular its recognition in those cases which are intractable to drugs. Our experience indicates that the localizatio of the responsible lesion may not be easy, for oug particular patient underwent many detailed clinical radiological, and E.E.G. investigations at sever悉 neurological centres before he was operated upon. The recognition of such cases depends mainly upoळ clinical observation, and the E.E.G. data may be normal or somewhat inconclusive. Indeed, if a provisional lateralization can be deduced from clinical or E.E.G. data, an exploratory craniotomy under local analgesia with all the facilities for electrocorticography and electrical stimulation of the brain may be warranted. It was only in this way that we were able to help our patient.

Finally we must remember that lesions in the region of the supplementary motor and sensory area may produce epileptic attacks characterized by similar tonic posturing to that seen in our patients but without the phenomenon of induction by movement (Kennedy, 1959). The distinction between such cases and cases of 'seizures induced by movement' may be premature until more extensive neurosurgical and pathological studies have been made.

\section{SUMMARY}

A case of 'epilepsy induced by movement' comparable to the group described recently under this title by Lishman et al. (1962) is reported. An exploratory craniotomy disclosed a small cortical cicatrix at the 
superior border of the left hemisphere $2 \mathrm{~cm}$. in front of the Rolandic fissure. Resection of the cicatrix resulted in subsequent complete relief of the symptoms. Lishman and his colleagues considered this condition to be rare, they did not know its pathological basis, and they did not report any patient who had been operated upon. It is suggested that other cases of such epilepsy may also have similar cortical lesions. Difficulties in diagnosis and localization are discussed, and the conclusion is drawn that should treatment with anticonvulsant drugs prove inadequate in such a case, an exploratory craniotomy may be warranted.

One of us (E.A.S.) is in receipt of a grant from the research funds of Guy's Hospital, and wishes to acknowledge his gratitude.

\section{REFERENCES}

Falconer, M. A., Driver, M. V., and Serafetinides, E. A. (1962). Brain, 85,521 .

Goldie, L., and Green, J. M. (1959). Ibid.. 82, 505.

Gowers, W. R. (1901). Epilepsy, 2nd ed. Churchill, London.

James, I. P. (1959). Personal communication.

Kennedy, W. A. (1959). Brain, 82, 147.

Lishman, W. A., Symonds, C. P., Whitty, C. W. M., and Willison, R. G. (1962). Ibid., 85, 93.

Michaux, L., and Granier (1945). Ann. méd. psychol., 103 (2), 172.

Mitchell, W., Falconer, M. A., and Hill, D. (1954). Lancet, 2, 626

Penfield, W., and Jasper, H. (1954). Epilepsy and the Functional Anatomy of the Human Brain. Little, Brown, Boston.

- , and Welch, K. (1951). A.M.A. Arch. Neurol. Psychiat., 66, 289. Pitha, V. (1938). Rev. neurol., 70, 178.

Sherwood, S. L., and 10 collected authors (1962). Arch. Neurol. (Chic.), 6, 49. 